\title{
Photoconductivity of Solution-Processed $\mathrm{MoS}_{2}$ Films
}

Graeme Cunningham, Umar Khan, Claudia Backes, Damien Hanlon, David

McCloskey, John Donegan and Jonathan N Coleman*

School of Physics, CRANN \& AMBER, Trinity College Dublin, Dublin 2, Ireland

*colemaj@tcd.ie

ABSTRACT: Solution-exfoliated $\mathrm{MoS}_{2}$ nano-platelets were formed into thin films by deposition onto a water surface followed by transfer to indium tin oxide coated glass. After drying, a gold electrode was evaporated on top to give a sandwich structure with quasi-Ohmic contacts. Illumination of this device with broadband light of $\sim 1 \mathrm{~kW} / \mathrm{m}^{2}$ intensity gave a fourfold increase in conductivity. The photocurrent increased sub-linearly with intensity and exponentially with time indicating the presence of traps. The photo-responsively was $\sim 10^{-4} \mathrm{~A} / \mathrm{W}$ at $15 \mathrm{~V}$, competitive with other 2-dimensional photoconductors. This work demonstrates the potential for liquidexfoliated, inorganic nanosheets to be fabricated into low-cost optoelectronic devices.

ToC entry
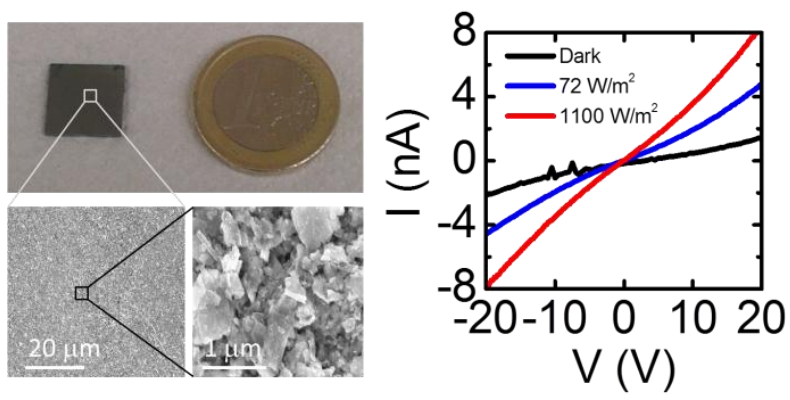

We have prepared solution-processed thin films of $\mathrm{MoS}_{2}$ nano-platelets which show four-fold conductivity increase under 1-sun illumination. 
Introduction

Over the last few years interest in 2-dimensional (2D) nano-materials has developed rapidly, partly due to the unprecedented success ${ }^{1}$ of graphene. Of the many types of $2 \mathrm{D}$ material, ${ }^{2}$ the family of transition metal dichalcogenides (TMDs) is particularly interesting. ${ }^{3,4}$ TMDs consist of 2D monolayers which are constructed from a plane of transition metal atoms, usually $\mathrm{Mo}, \mathrm{W}$ or $\mathrm{Nb}$, sandwiched between two planes of chalcogen atoms i.e. $\mathrm{S}$, Se or Te. Under normal circumstances, TMD monolayers are stacked together to form 3dimensional crystals. However, it has recently been shown that such crystals can be exfoliated to give mono- or few-layer flakes on surfaces. ${ }^{5}$ Alternatively, mono- or few-layerthick films can be grown directly by CVD. ${ }^{6}$ Monolayers of TMDs such as $\mathrm{MoS}_{2}$ have generated much interest due to their exceptional electronic and optical properties. ${ }^{3}$ For example $\mathrm{MoS}_{2}$ and $\mathrm{WS}_{2}$ monolayers are semiconductors and have been used as the basis of a wide range of electronic and optoelectronic devices including transistors, logic circuits, sensors and light emitting diodes. ${ }^{3,7}$ In particular, mono-and few-layer $\mathrm{MoS}_{2}$ and $\mathrm{WS}_{2}$ display photoconductivity and so may find applications as sensitive photodetectors. ${ }^{8-14}$ This is an application to which these materials appear to be particularly suited. For example, individual $\mathrm{MoS}_{2}$ monolayers have very high photosensitivity, up to $880 \mathrm{~A} / \mathrm{W}$ at low illumination power. ${ }^{10,12}$ This, coupled with their extremely low noise equivalent power, makes them ideal for very low level photo-detection. ${ }^{10,12}$ In addition, their spectral range can be tuned by control of the $\mathrm{MoS}_{2}$ flake thickness, ${ }^{15}$ leading to a broad photo-response which is expected to extend beyond $900 \mathrm{~nm}$ for thick $\mathrm{MoS}_{2}$ flakes. ${ }^{9}$ In addition, $\mathrm{MoS}_{2}$ photodetectors are extremely robust, working at high temperatures ${ }^{12}$ and in harsh environments with high radiation fluences. ${ }^{16}$

All of the work described above was performed on CVD-grown or mechanicallyexfoliated TMDs. Another way to produce 2D materials is to exfoliate layered materials in 
liquids. ${ }^{2}$ Liquid exfoliation has been demonstrated for graphite, ${ }^{17} \mathrm{TMDs}^{18}$ and a range of other layered crystals ${ }^{19}$ and is a cheap and simple way to produce large quantities of $2 \mathrm{D}$ materials. ${ }^{2}$ One big advantage of this process is that liquid exfoliation gives suspensions of 2D nanosheets which can easily be processed into composites, films or other structures. Such techniques have been used to prepare samples for a range of applications, mainly in electrochemistry. ${ }^{2,4}$ However, relatively few electronic or optoelectronic device applications have been demonstrated for liquid exfoliated nanosheets. ${ }^{3}$ This is unfortunate as devices based on such materials could be fabricated easily and cheaply using a range of techniques such as spraying ${ }^{20}$, inkjet printing ${ }^{21}$ or gravure coating ${ }^{22}$. Here we show that liquid exfoliation techniques can be used to give partially exfoliated, but solution processable $\mathrm{MoS}_{2}$ suspensions. These suspensions can be formed into thin films which, when electrically connected, show promising photoconductive properties.

Experimental procedure

The $\mathrm{MoS}_{2}$ starting powder was purchased from Sigma Aldrich $(<2 \mu \mathrm{m}$ particle size, product number 234842) and used as supplied. Nano-platelets of $\mathrm{MoS}_{2}$ were exfoliated and suspended in liquid using well-established solvent exfoliation techniques. ${ }^{2,17,18,23} \mathrm{MoS}_{2}$ powder $(1 \mathrm{~g})$ was added to HPLC-grade isopropanol (IPA, $100 \mathrm{ml})$ in a glass beaker. The sample was sonicated with a stand-mounted ultrasonic tip (Heilsher model UP200S, 200W, $24 \mathrm{kHz}$ ) for 24 hours at $60 \%$ amplitude in pulsed mode with $2 \mathrm{~s}$ on and $1 \mathrm{~s}$ off. This results in formation of a dispersion of $\mathrm{MoS}_{2}$ nano-platelets with a wide range of sizes and thicknesses.

When exfoliating layered materials in liquids, a centrifugation step is normally employed post sonication to remove the larger nano-platelets and any partially exfoliated crystallites. ${ }^{17,18,23}$ However, we found that films prepared from centrifuged $\mathrm{MoS}_{2}$ dispersions always displayed pinholes extending from top to bottom as evidenced by electrical shorting 
when electrodes were applied in a sandwich structure. Here, we avoid centrifugation as we found films of un-centrifuged $\mathrm{MoS}_{2}$ to be generally pinhole free. Shorting can occur in films consisting of discrete nano-scale entities, even when the films are relatively uniform and free of well-defined holes, due to diffusion of metal through the porous internal structure of the film. That shorting occurs for centrifuged films but not for non-centrifuged samples implies that the film microstructure depends strongly on the details of sample processing. Further work is needed to fully understand such effects. Because our dispersions were not centrifuged, they are expected to contain larger $\mathrm{MoS}_{2}$ nano-platelets and small crystallites as well as thin nanosheets. However, most importantly, they are still solution processable. To investigate the nature of the dispersed $\mathrm{MoS}_{2}$, we performed TEM characterisation (figure 1A) which showed the presence of micron sized nano-platelets. However, these were largely electron opaque suggesting the nano-platelets to be relatively thick. To measure the nanoplatelet thickness, we deposited a few drops of dispersion onto $\mathrm{Si} / \mathrm{SiO}_{2}$ (200 $\mathrm{nm}$ oxide thickness), just dried them in ambient air before imaging with AFM (figure 1B). Measurement of the thickness of many nano-platelets allowed statistical analysis (figure 1C) showing a broad distribution peaked around $50 \mathrm{~nm}$. This is consistent with the presence of both well- and poorly-exfoliated nano-platelets as expected. In addition, the lateral nanoplatelet dimensions were measured by TEM (figure 1D) and showed typical nano-platelets to be approximately $1 \mu \mathrm{m}$ in length.

These dispersions were used to form thin films for electrical testing. In order to avoid the formation of pinholes, a number of film formation methods were attempted before settling on a technique based in principle on the Langmuir-Blodgett method. A $250 \mathrm{ml}$ conical flask was filled to the brim with Millipore Water and, using a pipette, a few drops of the $\mathrm{MoS}_{2}$ dispersion were slowly dropped onto the rim of the flask and allowed to flow down into the water. This technique employs $\mathrm{MoS}_{2}$ 's inherent hydrophobicity: interaction with the water 
drives the $\mathrm{MoS}_{2}$ to the surface while the IPA enters the water subphase. The conical flask is ideal for keeping the concentration of IPA low in a larger body of water whilst allowing film formation over a smaller area. At first the $\mathrm{MoS}_{2}$ aggregates form at a number of independent nucleation sites. However, as more dispersion is added these sites coalesce resulting in the entire liquid surface becoming covered with a film of $\mathrm{MoS}_{2}$ nano-platelets.

To transfer this film onto a substrate, an indium tin oxide (ITO)-covered glass slide $(1.2 \mathrm{~cm} \times 1.2 \mathrm{~cm})$ was inserted below the surface using a set of tweezers and slowly pulled out at an angle of $\sim 75^{\circ}$ to the horizontal. This resulted in the ITO being coated with a thin film of $\mathrm{MoS}_{2}$ nano-platelets. This film was allowed to dry naturally in ambient air until appearing dry whereby it was then placed onto a hotplate at $250^{\circ} \mathrm{C}$ for 3 minutes before being removed and allowed to cool. The $\mathrm{MoS}_{2}$ film thickness was increased by re-dipping a number of times. However, the water had to be changed every 2-3 coatings to avoid excessive IPA content compromising the film formation.

Results and Discussion

The resultant films were dark grey and visually quite uniform (figure 1F). SEM examination (Zeiss Ultra Plus SEM) showed them to be continuous over length scales greater than a few microns (figure $1 \mathrm{G}$ ). However, when viewed at higher magnification it becomes clear that they are composed of a disordered array of nano-platelets of $\mathrm{MoS}_{2}$ (figure $1 \mathrm{H}$ ). The film for which we present electrical data below had a thickness of $\sim 6 \mu \mathrm{m}$ (achieved after 9 dips). However, the thickness varied by $\sim 20 \%$ over a line scan of $800 \mu \mathrm{m}$ (Dektak $6 \mathrm{M}$ Stylus Profiler) showing that this film formation method could certainly be improved. Raman spectra were recorded for such films (figure 1I). These were very similar to the equivalent spectra for the starting powder confirming the films to consist of relatively thick flakes (the Raman spectra of exfoliated $\mathrm{MoS}_{2}$ flakes approaches that of bulk $\mathrm{MoS}_{2}$ for flakes thicker than 
$\sim 6$ layers $).{ }^{24}$ We note the peak positions are slightly shifted from those expected for crystalline $\mathrm{MoS}_{2}$ as is sometimes observed for powdered samples. ${ }^{25}$ We also measured the absorbance spectrum of a thin layer prepared on a glass substrate by a single dipping cycle (figure 1J). This spectrum displays the features expected from $\mathrm{MoS}_{2}$ although the spectrum is probably superimposed on a scattering background. ${ }^{18}$

Devices were fabricated by evaporating a gold electrode $\left(\sim 50 \mathrm{~nm}\right.$ thick, $1.98 \mathrm{~cm}^{2}$ electrode overlap area) on top of the $\mathrm{MoS}_{2}$ film to give an ITO/MoS $/ \mathrm{Au}$ sandwich structure arrangement (figure $2 \mathrm{~A}$ inset). After evaporation, films were annealed in air at $200^{\circ} \mathrm{C}$ for 1 hour. Typically, such a mild anneal resulted in almost an order of magnitude current increase and a considerable reduction in current noise.

We expect this combination of ITO and gold electrodes to give roughly Ohmic contacts on both sides of the device. Bulk $\mathrm{MoS}_{2}$ is generally n-type with a band gap of 1.2 $\mathrm{eV}^{3}$ For bulk $\mathrm{MoS}_{2}$ (appropriate here because of the partial exfoliation) the conduction band edge, Fermi energy and valence band edge are at energies of 4.4, 4.8 and $5.6 \mathrm{eV}$ below the vacuum level. ${ }^{26}$ This means that the workfunction of the ITO $(\sim 4.5-4.8 \mathrm{eV})^{27}$ is expected to lie between the conduction band edge and the Fermi energy of $\mathrm{MoS}_{2}$. This is expected to result in an Ohmic contact. ${ }^{28}$ Although the work function of the gold does not lie between $\mathrm{MoS}_{2}$ 's Fermi level and conduction band edge, a number of papers have shown good electron injection at $\mathrm{Au} / \mathrm{MoS}_{2}$ interfaces, which is consistent with the presence of Ohmic contacts. ${ }^{29-32}$ In fact, detailed measurements have shown that the potential barrier at the $\mathrm{MoS}_{2} / \mathrm{Au}$ interface is extremely small. ${ }^{33}$ This may be partially explained by chemical bonding between gold and sulphur atoms, located at flake edges or defects, leading to a very low injection barrier and so a quasi-Ohmic contact. 
Shown in figure $2 \mathrm{~A}$ is an $\mathrm{I}-\mathrm{V}$ curve for an ITO/MoS2/Au sandwich structure device taken in the dark. This curve is symmetric around the origin, as expected if both contacts are Ohmic. $^{34}$ The dark conductivity is $\sim 2 \times 10^{-10} \mathrm{~S} / \mathrm{m}$, three orders of magnitude lower than measured for similar films but with in-plane current flow. ${ }^{23}$ This is consistent with the anisotropy between in-plane and out of plane conductivities in graphite and suggests the current to be limited by inter-flake transport. ${ }^{35}$

In this paper we are interested in the photoconductivity of films of $\mathrm{MoS}_{2}$ nanoplatelets. As such, we have studied the effect of illuminating the sample through the ITO with a full spectrum solar simulator (Newport 96000), fitted with a Xe arc discharge lamp (150W) and air mass (AM) 1.5 filter. The lamp was calibrated against a standard Si based reference cell. Light intensity at the sample was controlled using a set of neutral density filters (NDFs) purchased from Newport. Shown in figure 2A are I-V curves measured for an $\mathrm{MoS}_{2}$ film illuminated at different light intensities compared to the dark curve. All curves are symmetric about zero and linear at low voltage although some curvature is observed at higher voltages. Assuming the contacts are indeed Ohmic, such curvature is probably due to space charge effects. ${ }^{28}$ However, such behaviour will not be analysed in this work.

We measured the conductivity (measured in the -2 to $2 \mathrm{~V}$ range) as a function of light intensity, $\mathrm{F}$, as shown in figure $2 \mathrm{~B}$. The conductivity increases significantly from its dark value of $2 \times 10^{-10} \mathrm{~S} / \mathrm{m}$, reaching $8 \times 10^{-10} \mathrm{~S} / \mathrm{m}$ for $\mathrm{F}=1100 \mathrm{~W} / \mathrm{m}^{2}$. We note that this increase is not linear as is usually observed for semiconductors. ${ }^{28}$ Rather, the conductivity increase (i.e. the photoconductivity, $\sigma_{p h}$ ) scales roughly as $\sigma_{p h} \propto F^{\gamma}$ with $\gamma=0.5$. In the simplest case, such behaviour is usually associated with bimolecular recombination, ${ }^{28,36}$ which is usually observed in insulators where the density of optically generated carriers greatly exceeds that of free carriers in the dark. ${ }^{37}$ The majority of papers studying photoconductivity of individual 
monolayer (or few-layer) $\mathrm{MoS}_{2}$ or $\mathrm{WS}_{2}$ have observed linear (or near-linear) dependence of photocurrent on light intensity. ${ }^{8,9,13,14}$ However, a number of papers on such samples report sub-linear behaviour with $\gamma$ between 0.5 and $0.71 .{ }^{10-12}$ However, the materials studied in these papers are semiconductors with reasonable high dark carrier densities making bimolecular recombination unlikely. In such cases, sub-linear intensity dependence is likely to be associated with the presence of traps. ${ }^{28}$

Simple analyses of bimolecular recombination which predict $F^{1 / 2}$ behaviour generally assume trap free systems. ${ }^{28}$ However, in many real systems traps are present and manifest themselves through non-linear dependence of photocurrent on intensity and a relatively slow increase and decay of photocurrent with time. ${ }^{28}$ To test for the presence of traps, we measured the current as a function of time under constant bias of $15 \mathrm{~V}$. During the measurement, the light was switched on and later switched off. Examples of the resultant curves are shown in figure $2 \mathrm{C}$. In all cases, on illumination a fast photocurrent increase was observed followed by a slow photocurrent increase. Similarly, on cessation of illumination there is a fast decay component followed by a slow decay. Such a slow rise and decay of photocurrent are strong indicators of the presence of traps. ${ }^{28}$

Fast rise and decay of photocurrent has been observed by a number of authors in experiments on individual monolayer (or few-layer) $\mathrm{MoS}_{2}$ or $\mathrm{WS}_{2},{ }^{8,9,11,13}$ and usually in systems with linear intensity dependence that are assumed to be trap free. In addition, slow photocurrent rise and decay have also been observed. ${ }^{10,14}$ This has been attributed to traps associated with the interaction of the surface of the flake with either the substrate or the ambient gas. We note that a combination of fast and slow photocurrent rise and decay has not been reported for $\mathrm{MoS}_{2}$ or $\mathrm{WS}_{2}$. However, such behaviour is known for semiconductors such as n-type silicon. ${ }^{28,} 38$ In such behaviour, the fast component is attributed to the initial 
equilibration of carrier generation and recombination rates and occurs on the timescale of the carrier lifetime. This can be as fast as $\sim 1 \mathrm{~ns}$ in $\mathrm{MoS}_{2}$ and so is hard to measure experimentally. ${ }^{9}$ The slow photocurrent rise is associated with the effects of carrier trapping. ${ }^{28}$ Once the light is switched off, the fast decay is due to the recombination of free carriers while the slow decay represents the thermal detrapping of carriers.

As described above, the presence of traps leads to slow rise (decay) of photocurrent as traps fill (empty), slowing the approach to steady state behaviour. A mono-exponential rise (or decay) implies the presence of a single type of discrete trapping state within the gap where re-trapping of freed carriers is negligible. ${ }^{28,39-41}$ The dark current on decay can be slightly higher than before illumination. This persistent photoconductivity (PPC) is a common effect and is associated with very deep traps. ${ }^{40,42,43}$

For all light intensities these slow photocurrent increases can be fit to an exponential function of the form

$$
\Delta I_{R}=\Delta I_{f, R}+\Delta I_{s, R}\left(1-e^{-t / \tau_{R}}\right)
$$

where $\Delta I_{f, R}$ is the fast component of the rising photocurrent, $\Delta I_{s, R}$ the slow component of the rising photocurrent and $\tau_{\mathrm{R}}$ the time constant. Similarly, the slow photocurrent decay can be fit to

$$
\Delta I_{D}=\Delta I_{s, D} e^{-t / \tau_{D}}+\Delta I_{p, D}
$$

where $\Delta I_{s, D}$ is the slow component of the decaying photocurrent, $\tau_{\mathrm{R}}$ is the time constant and $\Delta I_{p, D}$ is the persistent photocurrent. ${ }^{28}$ Note, the fast component (i.e. $\Delta I_{f, D}$ ) of the decaying photocurrent can be read off the graph. 
The fast components of photocurrent rise and decay have been plotted against $\mathrm{F}$ in figure $2 \mathrm{D}$. In both cases they sit on the same line consistent with $\Delta I_{f} \propto F^{\gamma}$ where $\gamma=0.54$. While this is very close to the 0.5 expected for bimolecular recombination care must be taken as the relationship between this fast component and the trap distribution is not clear. Indeed power-law behaviour whose exponent is controlled solely by the details of the trap distribution is well known. ${ }^{28}$

The slow component of the rise and decay of photocurrent was also plotted versus $\mathrm{F}$ in figure 2E. Both $\Delta I_{s, R}$ and $\Delta I_{s, D}$ behave similarly with no obvious correlation to light intensity. This behaviour is not surprising as the slow component is thought to be controlled by the trap density. ${ }^{28}$ The PPC is not plotted but was close to $0.5 \mathrm{nA}$ for all samples.

We note that after long illumination times, the photocurrent will saturate at $\Delta I_{R, s a t}=\Delta I_{f, R}+\Delta I_{s, R}$. This saturation photocurrent increases weakly with intensity. As a result, the saturation photoresponsivity (usually defined as $P_{R}=\Delta I_{R, s a t} / F$ where $\mathrm{F}$ is the incident intensity) falls with increasing intensity. However, at low intensity $\mathrm{P}_{\mathrm{R}} \approx 10^{-4} \mathrm{~A} / \mathrm{W}$ (measured at $15 \mathrm{~V}$ ), similar to both the lower end of the range reported for CVD grown TMDs $^{11}$ and to early graphene photodetectors. ${ }^{44}$ However, we note that this does not take the wavelength dependence of the photoconductivity into account. To test this we measured the action spectrum of the photoconductive response using a set of notch filters and the solar simulator. Because the photocurrent scales roughly as $F^{1 / 2}$, we calculate the measured photocurrent divided by the square root of the integrated incident intensity. This is plotted versus wavelength in figure $1 \mathrm{~J}$ (inset). We find the spectral response to increase with decreasing wavelength as expected from the absorbance spectrum. We see an apparent peak around $650 \mathrm{~nm}$ consistent with the A exciton position in $\mathrm{MoS}_{2} \cdot{ }^{45}$ In addition, the spectral response increases sharply below $500 \mathrm{~nm}$ as observed previously for $\mathrm{MoS}_{2}$ monolayers. ${ }^{10}$ 
This suggests that the photoresponsivity calculated using the absorbed power would be considerably higher than the value quoted above.

In figure $2 \mathrm{~F}$ time constants for the rise and decay of photocurrent are plotted against $\mathrm{F}$ and tend to fall off as roughly $\mathrm{F}^{-1 / 2}$. It has been shown that when the $\tau_{\mathrm{D}}$ scales with intensity as $\tau_{D} \propto F^{-\gamma}$, this means the observed time constant equals the carrier lifetime (including time spent in traps). ${ }^{46}$ That this lifetime is so long indicates that trapping is a problem in these films. The main difference between the films used here and the $\mathrm{MoS}_{2}$ or $\mathrm{WS}_{2}$ samples previously studied is that these films consist of a disordered array of nano-platelets. It is entirely possible that states associated with flake edges are acting as traps. Future work will study methods of passivation of the flake edges with the aim of reducing the number of traps and so minimising their effect.

\section{Conclusion}

In summary, we have demonstrated a method to prepare solution-processed thin films of $\mathrm{MoS}_{2}$ which can be fabricated into photodetectors. These films show a four-fold increase in conductivity on exposure to $\sim 1 \mathrm{~kW} / \mathrm{m}^{2}$ broadband excitation. However, due to the presence of traps the photo-response scales sub-linearly with intensity and increases slowly with time. However, the photo-responsivity is reasonable, matching that of early monolayer graphene reports. The ability to prepare photo-detectors from inorganic nano-materials such as $\mathrm{MoS}_{2}$ by solution processing techniques represents an important step forward. Such techniques allow the preparation of cheap, large area devices quickly and at low cost. We consider this the first step toward processing solution-exfoliated 2D materials into a range of optoelectronic devices.

We acknowledge the European Research Council grant SEMANTICS for financial support. 

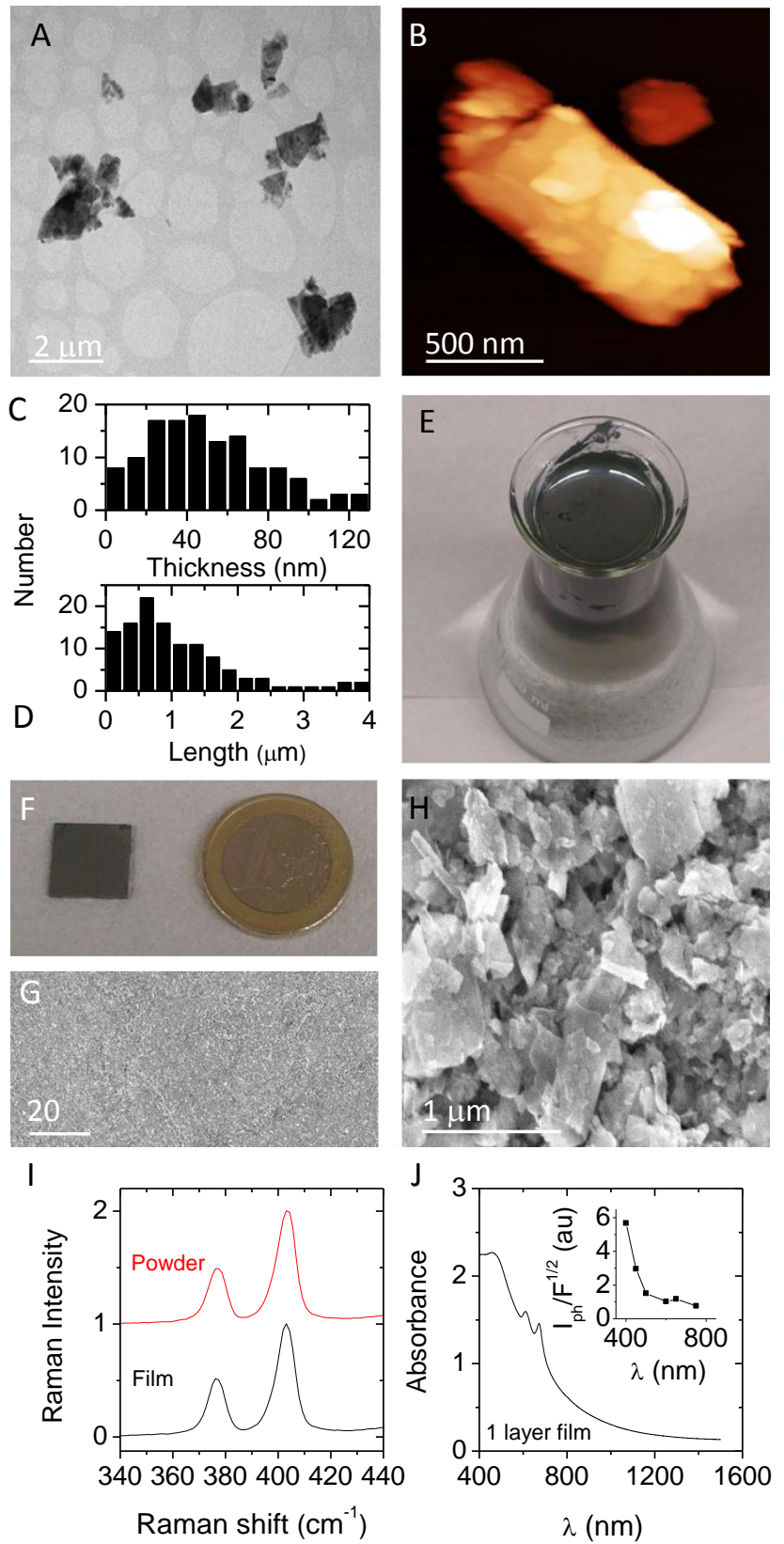

Figure 1: A) TEM image of solution processed $\mathrm{MoS}_{2}$ nano-platelets. B) AFM image of an $\mathrm{MoS}_{2}$ nano-platelet deposited on a substrate. C) Thickness distribution for $\mathrm{MoS}_{2}$ nanoplatelets deposited on a $\mathrm{Si} / \mathrm{SiO}_{2}$ substrate as measured by AFM. D) Length distribution as measured by TEM. E) Conical flask containing water with a film of $\mathrm{MoS}_{2}$ nano-platelets floating on top. F) Photograph of a film prepared using 9 dipping cycles. G) Widefield and H) close-up SEM images of a film of $\mathrm{MoS}_{2}$ nano-platelets. I) Raman spectra of both starting 
powder and film prepared from an uncentrifuged $\mathrm{MoS}_{2}$ dispersion. J) Optical absorbance spectrum of an $\mathrm{MoS}_{2}$ film prepared by a single dipping cycle. Inset: Action spectrum for a film prepared from 9 dipping cycles. The photoconductive behaviour is represented by the photocurrent divided by the square root of the integrated incident intensity (because $\sigma_{p h} \propto F^{\gamma}$ with $\left.\gamma=0.5\right)$
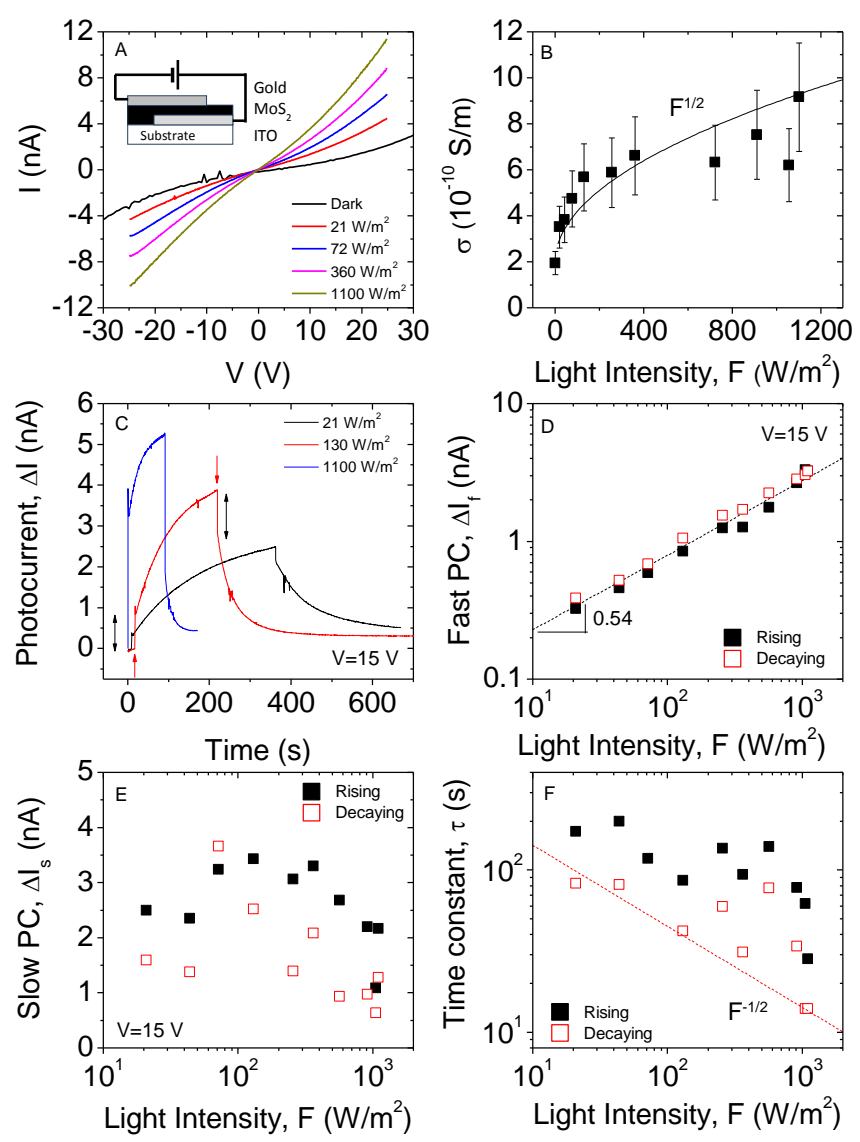

Figure 2: A) IV curves for an $\mathrm{MoS}_{2}$ film in the dark and under various light intensities, F. Inset: Electrode structure. B) Measured conductivity as a function of light intensity. The line illustrates square root behaviour. C) Photocurrent, PC, (dark current removed) plotted versus time for three different light intensities $(\mathrm{V}=15 \mathrm{~V})$. For the red curve, the red arrows indicate 
the times the light was switched on and off while the black double-headed arrows illustrate the fast portions of the rise and decay. D) Fast components of both rise and decay of photocurrent plotted versus light intensity. The dashed line is a power law fit. E) Slow components of both rise and decay of photocurrent plotted versus light intensity. F) Time constants for both rising and decaying photocurrents plotted versus light intensity. The dashed line represents $\mathrm{F}^{-1 / 2}$ behaviour. 


\section{References}

1. A. K. Geim, Science, 2009, 324, 1530-1534.

2. V. Nicolosi, M. Chhowalla, M. G. Kanatzidis, M. S. Strano and J. N. Coleman, Science, 2013, 340, 1226419.

3. Q. H. Wang, K. Kalantar-Zadeh, A. Kis, J. N. Coleman and M. S. Strano, Nature Nanotechnology, 2012, 7, 699-712.

4. M. Chhowalla, H. S. Shin, G. Eda, L. J. Li, K. P. Loh and H. Zhang, Nat. Chem., 2013, 5, 263-275.

5. K. S. Novoselov, D. Jiang, F. Schedin, T. J. Booth, V. V. Khotkevich, S. V. Morozov and A. K. Geim, Proceedings of the National Academy of Sciences of the United States of America, 2005, 102, 10451-10453.

6. $\quad$ Y. J. Zhan, Z. Liu, S. Najmaei, P. M. Ajayan and J. Lou, Small, 2012, 8, 966-971.

7. $\quad$ R. S. Sundaram, M. Engel, A. Lombardo, R. Krupke, A. C. Ferrari, P. Avouris and M. Steiner, Nano Letters, 2013, 13, 1416-1421.

8. C. Ballif, M. Regula, F. Levy, F. Burmeister, C. Schafle, T. Matthes, P. Leiderer, P. Niedermann, W. Gutmannsbauer and R. Bucher, Journal of Vacuum Science \& Technology aVacuum Surfaces and Films, 1998, 16, 1239-1243.

9. W. Choi, M. Y. Cho, A. Konar, J. H. Lee, G.-B. Cha, S. C. Hong, S. Kim, J. Kim, D. Jena, J. Joo and S. Kim, Advanced Materials, 2012, 24, 5832-5836.

10. O. Lopez-Sanchez, D. Lembke, M. Kayci, A. Radenovic and A. Kis, Nature Nanotechnology, 2013, 8, 497-501.

11. N. Perea-López, A. L. Elías, A. Berkdemir, A. Castro-Beltran, H. R. Gutiérrez, S. Feng, R. Lv, T. Hayashi, F. López-Urías, S. Ghosh, B. Muchharla, S. Talapatra, H. Terrones and M. Terrones, Advanced Functional Materials, 2013, Online publication, DOI: 10.1002/adfm.201300760.

12. D.-S. Tsai, K.-K. Liu, D.-H. Lien, M.-L. Tsai, C.-F. Kang, C.-A. Lin, L.-J. Li and J.-H. He, Acs Nano, 2013, 7, 3905-3911.

13. Z. Y. Yin, H. Li, L. Jiang, Y. M. Shi, Y. H. Sun, G. Lu, Q. Zhang, X. D. Chen and H. Zhang, Acs Nano, 2012, 6, 74-80.

14. W. J. Zhang, J.-K. Huang, C.-H. Chen, Y.-H. Chang, Y.-J. Cheng and L.-J. Li, Advanced Materials, 2013, 25, 3456-3461.

15. H. S. Lee, S. W. Min, Y. G. Chang, M. K. Park, T. Nam, H. Kim, J. H. Kim, S. Ryu and S. Im, Nano Letters, 2012, 12, 3695-3700.

16. D.-S. Tsai, D.-H. Lien, M.-L. Tsai, S.-H. Su, K.-M. Chen, J.-J. Ke, Y.-C. Yu, L.-J. Li and J.H. He, The IEEE Journal of Selected Topics in Quantum Electronics 2014, 20, 3800206.

17. Y. Hernandez, V. Nicolosi, M. Lotya, F. M. Blighe, Z. Y. Sun, S. De, I. T. McGovern, B. Holland, M. Byrne, Y. K. Gun'ko, J. J. Boland, P. Niraj, G. Duesberg, S. Krishnamurthy, R. Goodhue, J. Hutchison, V. Scardaci, A. C. Ferrari and J. N. Coleman, Nature Nanotechnology, 2008, 3, 563-568.

18. J. N. Coleman, M. Lotya, A. O'Neill, S. D. Bergin, P. J. King, U. Khan, K. Young, A. Gaucher, S. De, R. J. Smith, I. V. Shvets, S. K. Arora, G. Stanton, H. Y. Kim, K. Lee, G. T. Kim, G. S. Duesberg, T. Hallam, J. J. Boland, J. J. Wang, J. F. Donegan, J. C. Grunlan, G. Moriarty, A. Shmeliov, R. J. Nicholls, J. M. Perkins, E. M. Grieveson, K. Theuwissen, D. W. McComb, P. D. Nellist and V. Nicolosi, Science, 2011, 331, 568-571.

19. M. Osada and T. Sasaki, Journal of Materials Chemistry, 2009, 19, 2503-2511.

20. V. Scardaci, R. Coull, P. E. Lyons, D. Rickard and J. N. Coleman, Small, 2011, 7, 2621-2628.

21. F. Torrisi, T. Hasan, W. P. Wu, Z. P. Sun, A. Lombardo, T. S. Kulmala, G. W. Hsieh, S. J. Jung, F. Bonaccorso, P. J. Paul, D. P. Chu and A. C. Ferrari, Acs Nano, 2012, 6, 2992-3006.

22. J. Puetz and M. A. Aegerter, Thin Solid Films, 2008, 516, 4495-4501.

23. G. Cunningham, M. Lotya, C. S. Cucinotta, S. Sanvito, S. D. Bergin, R. Menzel, M. S. P. Shaffer and J. N. Coleman, Acs Nano, 2012, 6, 3468-3480.

24. C. Lee, H. Yan, L. E. Brus, T. F. Heinz, J. Hone and S. Ryu, ACS Nano, 2010, 4, 2695-2700.

25. B. C. Windom, W. G. Sawyer and D. W. Hahn, Tribology Letters, 2011, 42, 301-310. 
26. T. Shimada, F. S. Ohuchi and B. A. Parkinson, Japanese Journal of Applied Physics Part 1Regular Papers Short Notes \& Review Papers, 1994, 33, 2696-2698.

27. M. Shanmugam, T. Bansal, C. A. Durcan and B. Yu, Applied Physics Letters, 2012, 101.

28. R. H. Bube, Photoconductivity of Solids, Wiley, 1960.

29. B. Radisavljevic, A. Radenovic, J. Brivio, V. Giacometti and A. Kis, Nature Nanotechnology, 2011, 6, 147-150.

30. D. Lembke and A. Kis, Acs Nano, 2012, 6, 10070-10075.

31. B. Radisavljevic, M. B. Whitwick and A. Kis, Acs Nano, 2011, 5, 9934-9938.

32. B. Radisavljevic, M. B. Whitwick and A. Kis, Applied Physics Letters, 2012, 101.

33. Personal communication from A. Kis, 2013.

34. J. Mort and D. M. Pai, Photoconductivity and related phenomena, Elsevier Scientific Pub. Co., 1976.

35. X. Tian, M. E. Itkis, E. B. Bekyarova and R. C. Haddon, Scientific Reports, 2013, 3, 1710.

36. E. Danielson, Z.-E. Ooi, C. J. Lombardo and A. Dodabalapur, Applied Physics Letters, 2013, 102, 173304.

37. R. K. Pal, S. Yadav, A. K. Agnihotri, D. Kumar and A. Kumar, Journal of Non-Oxide Glasses Vol, 2009, 1, 285-291.

38. J. R. Haynes and J. A. Hornbeck, Physical Review, 1955, 100, 606-615.

39. J. Wu, H. Li, Y. Liu and C. Xie, Journal of Applied Physics, 2011, 110, 123513.

40. A. Rose, Concepts in photoconductivity and allied problems, Interscience Publishers, 1963.

41. Y. Jiang, W. J. Zhang, J. S. Jie, X. M. Meng, X. Fan and S. T. Lee, Advanced Functional Materials, 2007, 17, 1795-1800.

42. A. S. Maan and D. R. Goyal, Optoelectronics and Advanced Materials, 2007, 1, 430-435.

43. M. Salis, A. Anedda, F. Quarati, A. J. Blue and W. Cunningham, Journal of Applied Physics, 2005, 97, 033709.

44. F. N. Xia, T. Mueller, Y. M. Lin, A. Valdes-Garcia and P. Avouris, Nature Nanotechnology, 2009, 4, 839-843.

45. J. A. Wilson and A. D. Yoffe, Advances in Physics, 1969, 18, 193-\&.

46. G. J. Adriaenssens, S. D. Baranovskii, W. Fuhs, J. Jansen and Ö. Öktü, Physical Review B, 1995, 51. 